\title{
Vertebral Compression Fracture with Intravertebral Vacuum Cleft Sign: Pathogenesis, Image, and Surgical Intervention
}

\author{
Ai-Min Wu, Yong-Long Chi, Wen-Fei Ni \\ Department of Orthopaedic Surgery, Second Affiliated Hospital of Wenzhou Medical College, Wenzhou, China
}

\begin{abstract}
The intravertebral vacuum cleft (IVC) sign in vertebral compression fracture patients has obtained much attention. The pathogenesis, image character and efficacy of surgical intervention were disputed. Many pathogenesis theories were proposed, and its image characters are distinct from malignancy and infection. Percutaneous vertebroplasty (PVP) or percutaneous kyphoplasty (PKP) have been the main therapeutic methods for these patients in recent years. Avascular necrosis theory is the most supported; PVP could relieve back pain, restore vertebral body height and correct the kyphotic angulation (KA), and is recommended for these patients. PKP seems to be more effective for the correction of KA and lower cement leakage. The Kümmell's disease with IVC sign reported by modern authors was incomplete consistent with syndrome reported by Dr. Hermann Kümmell.
\end{abstract}

Keywords: Intravertebral vacuum cleft; Pathogenesis; Vertebroplasty; Kyphoplasty; Kümmell's disease

\section{Introduction}

With the aging population, the incidence of osteoporotic vertebral compression fractures (OVCFs) is increasing and becoming a major health-care issue $[1,2]$. The intravertebral vacuum cleft (IVC) sign in these patients has obtained much attention in recent reports [3-5].

In flexion and extension lateral films, the IVC sign is characterized as dynamic instability or pseudoarthrosis [6,7]. Patients with IVC sign complained of severe pain, especially when they changed positions, and did not respond to bed rest, medication, corset and other conservative treatments. Thus, surgical intervention was recommended [8].

The IVC sign was first reported by Maldague et al. [9] as a sign of ischemic vertebral collapse, then Theodorou
[10] and Sarli et al. [11] described the details of this sign. On radiograph, it appears as a transverse, linear or semi-lunar radiolucent shadow. There were many different synonyms for this sign: such as "intravertebral cleft" [5], "linear intravertebral vacuum" [12], "intravertebral vacuum phenomenon" [13], "intravertebral vacuum sign" [14], "intraosseous vacuum phenomenon" [15], "intravertebral pseudarthrosis" [7] or "Kümmell disease" [16].

It was also reported that the IVC sign could be caused by long-term corticosteroid therapy, diabetes, arteriosclerosis, alcoholism, pancreatitis, radiation therapy, acute trauma, malignancy, and infection. Since the IVC sign caused by malignancy and infection have different pathogenesis and radiological features [9], the IVC sign caused by malignancy and infection were excluded from our study.

Received Apr 28, 2012; Revised Aug 22, 2012; Accepted Sep 13, 2012

Corresponding author: Yong-Long Chi

Department of Orthopaedic Surgery, Second Affiliated Hospital of Wenzhou Medical College,

109\# XueYuan Western Road, Wenzhou, Zhejiang, 325027, China

Tel: +86-0577-88002814, Fax: +86-0577-88002823, E-mail: Spinechi@163.com 


\section{Pathogenesis of IVC}

The pathogenesis of IVC was unclear, Maldague et al. [9] first associated avascular necrosis with IVC sign in 1978, they followed up 10 patients with an IVC sign range from one to six years. The histological data of one patient and radiological data of six patients were collected. They presumed that IVC was a specific sign of local bone ischemia. This avascular necrosis theory was also supported by anatomical studies: the vasculature of thoracic and lumbar vertebral bodies arises from paired segmental arteries, posterior central branches to the dorsum of the body supply two adjacent vertebral bodies, while anterior central branches to the ventral of the body supply one vertebral body. Therefore, the blood of the vertebral ventral zone only supplied by the short, branch early anterior central branches, putting theoretical higher risk of vascular insufficiency for the vertebral ventral zone. The weak blood supply of vertebral ventral zone was also proved by Ratcliffe [17].

Van Eenenaam and el-Khoury [18] reported a patient had a normal computed tomography (CT) scan and plain film at 3 weeks after initial back injury, while the bone scan showed an increased radionuclide uptake. The vertebral body was collapsed with IVC sign 9 weeks after injury. They considered bone scan as a sensitive imaging tool for early diagnosis of ischemic necrosis and they supposed that the avascular necrosis at 3 weeks after injury caused vertebral body collapse afterwards. Hermann et al. [19] reported a Gaucher's disease type 1 patient exhibiting IVC sign in the vertebral body, and the accumulation of glycosyl ceramide in Gaucher's cells could involve bony artery; therefore, this case could be additional evidence for avascular necrosis theory. The avascular necrosis theory was also supported by Bhalla and Reinus [12], and other authors [20].

Not all of the authors agree with the avascular necrosis theory as the pathogenesis of IVC. Kim et al. [3] analyzed 67 patients with IVC sign and noted that the IVC sign occurred predominantly in the thoracolumbar junction (81\%). For the wide activity and great load of the thoracolumbar junction region, they concluded that biomechanics also played an important role in the pathogenesis of IVC sign. According to recent literatures, osteoporotic vertebral fractures with or without IVC sign most commonly occur in the region of the thoracolumbar junction, consistent with the biomechanics theory. Osteoporosis was also considered as a factor that put the higher ratio ischemia of the vertebral body, because of decreased osteoblasts of these patients [21]. The study also found the significant inverse correlation between the bone mineral density (BMD) and the frequency of IVC sign [22].

Armingeat et al. [23] prospectively evaluated the presence of radiological findings regarding 15 patients with IVC over a 6-year period. He noted that 13 of them had intradiscal vacuum cleft adjacent to the fractured endplate, and communication between the intradiscal and intravertebral collections was observed in 5 patients. They suggested that the gas may migrate from the disk to the vertebral body, and the gas contained about $90 \%$ to $92 \%$ nitrogen.

\section{Kümmell's Disease or Not?}

Kümmell's disease was described by Dr. Hermann Kümmell [24] in 1895, characteristized as initially asymptomatic for weeks to months, and delayed post-traumatic osteonecrosis. It was divided into 3 stages: 1) Initial injury to the spinal column attended with a varying degree of surgical shock; 2) Relative comfort in which the patient is able to carry on his occupation; 3) Several weeks or months after injury, or even two or three years, an angular kyphosis develops with recurrence of pain, which is either localised over the spine or radiates over the extremities [25]. Moreover, Hosford [26] and Steel [27] described Kümmell's disease as five stages.

The diagnosis of Kümmell's disease was completely dependent on the clinical presentations at that time, lacking objective test evidence before the discovery of radiography. The clinical presentations of some patients with IVC sign were so similar to Kümmell's disease, and some authors regarded the IVC sign as "Kümmell's sign", patients with IVC sign were diagnosed as Kümmell's disease [2830], arousing fierce controversy.

Young et al. [31] argued that Kümmell's disease is distinguished from the typical osteoporotic compression fracture with IVC sign. In 2002, they reviewed the English language literatures since the 1950s and felt that only 5 cases met the diagnostic criteria set out by Dr. Hermann Kümmell. With the same view held by Swartz and Fee [16], they argued that eponymous diagnosis should be restricted to those individuals whose X-ray imaging around the time of the trauma was unremarkable, but later developed vertebral body collapse, and concluded 
that there were at most 10 Kümmell's disease patients in the past 30 years of literatures.

In fact, as reported by Hosford [26]: Kümmell's diseases was not rare as Kümmell first described six cases in his paper while other authors had reported many cases since 1895. Another fact was that Verneuil had described the same syndrome in 1892, which was earlier than Kümmell. In some instances it had been named as "KümmellVerneuil disease". The diagnosis of Kümmell's disease only based on clinical presentation, pathogenesis and imaging characteristics of Kümmell's disease were not described by Dr. Hermann Kümmell originally. As we know, medicine is the process of exploration. With the improvement of diagnostic methods, the standard diagnosis of diseases trend to based on etiological evidence. The pathogenesis and imaging character of Kümmell's disease that described by Dr. Hermann Kümmell originally was unclear, it cannot exclude the possibility that different etiologies between six patients reported by Dr. Hermann Kümmell and the Kümmell's disease reported by other following authors. The clinical symptoms of some patients with IVC sign were very similar to Kümmell's diseases, thus it

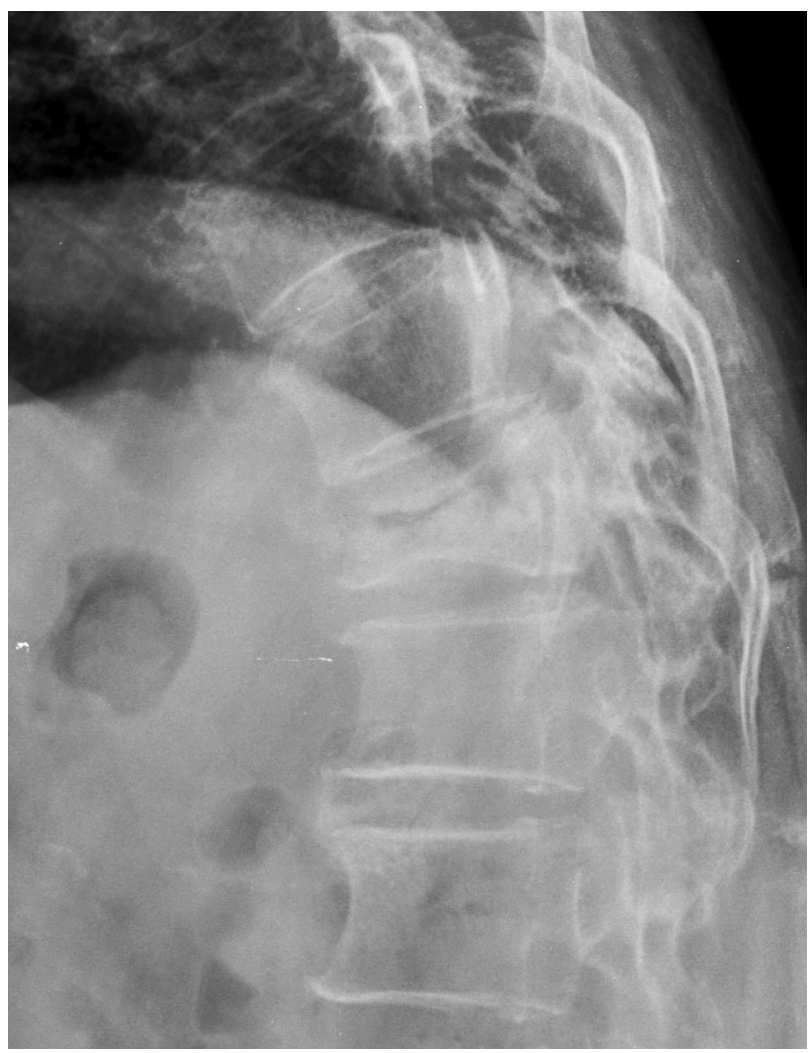

Fig. 1. The intravertebral vacuum cleft sign appears as a transverse, linear or semi-lunar radiolucent shadow in plain radiographs. is reasonable for some modern authors to regard the IVC sign as "Kümmell's sign", and diagnose some OVCFs patients with IVC sign as Kümmell's disease.

If vertebral avascular osteonecrosis with IVC caused by osteoporosis or corticosteroid therapy or other conditions were excluded, the real existence of Kümmell's disease will be questioned. No one can be sure if Kümmell's disease is reality or a myth. There are only two choices: call the vertebral avascular osteonecrosis with IVC as Kümmell' disease or let the name of Kümmell's disease disappear from textbooks. I believe we will change the former to commemorate the great Dr. Hermann Kümmell.

\section{Images of the IVC Sign}

After Maldague et al. [9] reported six cases who exhibited the IVC sign in radiographs, many studies described the details of this sign $[10,11,20,32]$, To sum up: 1 ) The IVC sign appears as a transverse, linear or semi-lunar radiolucent shadow in plain radiographs (Fig. 1). In addition, lateral views may help distinguish between an intraosseous, intervertebral, or bowel location of the lucency; the supine lateral views are more efficient to detect the IVC sign; 2) The sign could also be seen on CT scans, and appears more heterogeneous and irregular (Fig. 2);3) On magnetic resonance (MR) images, the sign is generally seen as low signal intensity on T1-weighted images, low signal intensity or high signal intensity on T2-weighted

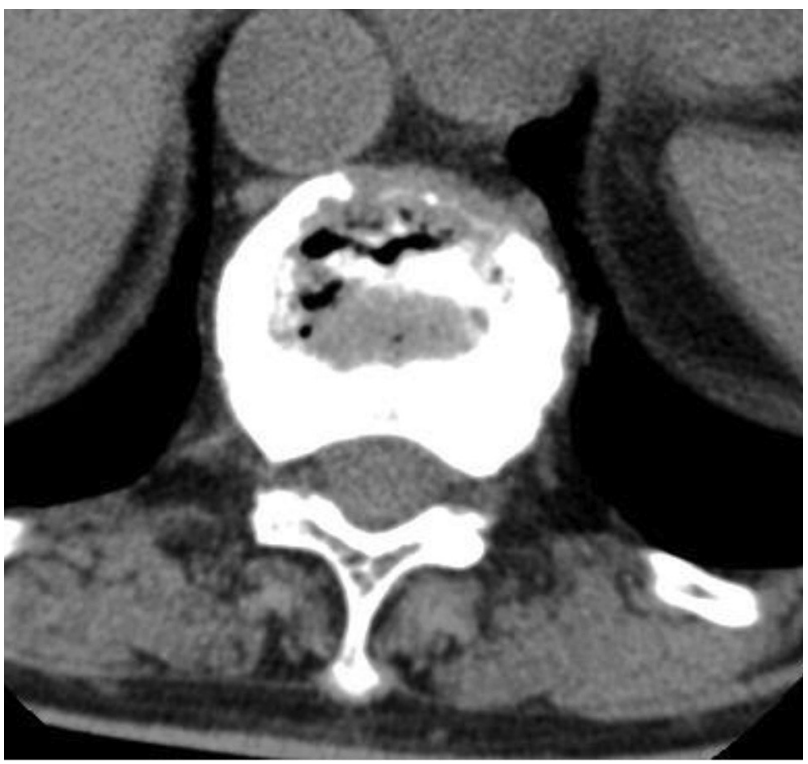

Fig. 2. The intravertebral vacuum cleft sign appears more heterogeneous and irregular on computed tomographic scan. 
images depending on the content of gas or liquid in cleft.

For patients with an acute osteoporotic fracture, fluid will fill the cleft from the adjacent bone marrow edema when patients are in the supine position just for 1 or 2 minutes [4]. In some cases, fluid was initially already present at the beginning of the MR examination. For patients with a fracture history of more than 3 months, the time of fluid collection is more than 20 minutes [33]. Therefore, patients with an acute fracture often show high signal intensity on T2-weighted images while patients with a longer fractured history often show lower signal intensity, because it is not enough time for fluid collection before MR examination.

The typical IVC sign is distinguished from the representation of malignancy in plain radiography, CT or MRI $[12,34]$, but they are often inadequate in distinguishing the distinction between malignancy and benign compression fractures. Thus, biopsy is needed for further diagnosis.

\section{Surgical intervention}

Patients with IVC sign usually have severe back pain and do not respond to conservative treatments such as bed rest, medication and orthosis. Surgical intervention is recommended [8]. Percutaneous vertebroplasty (PVP) is the main method used for these patients $[5,32,35]$, and percutaneous kyphoplasty (PKP) is also reported by some authors [36,37].
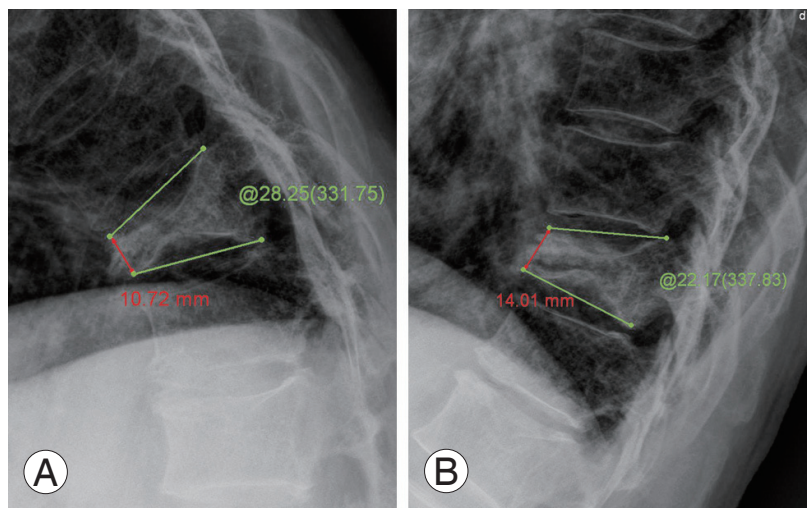

Fig. 3. The intravertebral vacuum cleft sign is regarded as pseudarthrosis, the anterior vertebral height and kyphotic angulation are changed on dynamic lateral flexion (A) and extension (B) views.

\section{Pain relief}

Chen et al. [38] reported 27 patients with IVC sign, all of them had content pain relief immediately after PVP, and Krauss et al. [39] reported that patients with IVC sign had the same pain reduction as patients without it. The same result was reported by Lane et al. [5]. Jang et al. [8] noted that 8 in 16 patients had complete pain relief, while 6 patients had moderate pain relief and 2 patients had no significant pain relief. This concludes that PVP is a reasonable procedure for these patients.

However, not all studies shared an optimistic view of PVP. Ha et al. [40] reported that the pain reduction of patients with IVC after PVP was less than those without cleft. Furthermore, Peh et al. [41] reported that 4 cases in 18 patients and Wiggins et al. [42] reported that 5 cases in 15 patients had no improvement or even aggravated back pain after PVP. The explanation of inadequate pain relief may be a predominant filling of the IVC, and the remaining vertebral body will remain unsupported and untreated. Most previous studies treat patients with IVC sign by PVP, and few authors manage them with PKP. The pain relief after PKP was not significantly different with PVP [37].

\section{Correction of kyphotic angulation}

PVP could correct kyphotic angulation (KA) of IVC patients more effectively than those without IVC sign $[32,39,43]$. The IVC was similar to pseudarthrosis (Fig. 3), and auto-reduction was obtained when patients were in the prone position. Preoperative kyphosis was reported as another influential factor regarding the degree of kyphosis correction after operation [44]. Preoperative dynamic instability seemed to be a good indicator of the potential to restore vertebral height $[6,45]$. PKP was reported to be better at correction of KA and restoration of vertebral height for patients with IVC sign $[37,46]$. In vitro study [47] likewise found that height was better restored after kyphoplasty compared to vertebroplasty.

Spinal deformation was related to functional limitation [48]. Thoracic compression fractures and kyphosis were related to pulmonary function decreases and pulmonary deaths [49]. Spinal deformity was also speculated as an independent risk factor of hip fracture [50]. These findings suggested that restoration of vertebral body height and correction of KA is an important component. How- 
ever, Kim et al. [35] reported that KA correction of $>5^{\circ}$ could be a poor prognostic factor. They presumed that patients with too much correction of KA resulted in more instability of the fractured segment, more paravertebral soft tissue or ligament injury, and had persistent pain in the first month after operation.

\section{Cement leakage and pulmonary embolism}

A comparative study [40] showed that cement leakage after PVP was observed in 9 out of 12 vertebrae cases with IVC sign (75\%), and higher than 24 cases in 58 vertebrae without it (32.6\%). Likewise, Nieuwenhuijse et al. [51] identified the IVC sign on MRI as additional strong risk factors of cement leakage. However, Krauss et al. [39] reported that the incidence of cement leakage was lower for vertebral bodies with IVC sign than those without it. Another comparative study [52] found no statistically significant difference regarding the incidence of cement leakage between vertebrae with IVC (53 of 107 vertebrae, 49.5\%) and those without IVC (78 of 193 vertebrae, 40.4\%) after PVP. The result was consistent with that of Jang et al. [8]. Wang et al. [37] reported that the incidence of cement leakage was lower for IVC patients after PKP, the lower pressure cavity created by PKP permits cement injected readily, and lowers the incidence of leakage.

The cement leakage ratio was not only influenced by the existence of IVC sign, but was also influenced by factors such as the viscosity of cement, the velocity and volume of injection, the inner pressure and the morphology of fractured vertebrae [51,53]. In the majority of cases, cement leakage is harmless and requires no further therapy. But caution should be taken to avoid intervertebral disk leakage and pulmonary cement embolism.

Tanigawa et al. [52] noted that the cement leakage into the intervertebral disk was significantly more frequent in vertebrae with IVC sign. The IVC and intradiscal vacuum cleft often co-exist, and a communication channel could be observed between them [23]. Cement was readily leaking into intervertebral disk via the channel. Intervertebral disk cement leakage is often asymptomatic, however, it was reported as one risk factor of new fracture at adjacent vertebrae $[54,55]$. The postoperative radiograph of a typical patient demonstrating the coexistence of IVC and intradiscal vacuum cleft in our department showed cement leak into the intervertebral disk, and an adjacent vertebral body fracture at 3 months after the first operation (Fig. 4).

Since X-rays and CTs were not conventionally performed on patients after PVP or PKP, and most pulmonary embolism patients were asymptomatic, many pulmonary cement embolisms remained undetected. A literature review demonstrated that the risk of pulmonary embolism ranged from $3.5 \%$ to $23 \%$ [56]. Although asymptomatic patients with a peripheral pulmonary cement embolism required no treatment besides clinical follow-up, sometimes the pulmonary cement embolism demonstrated fatal complications [57]. The study also indicated [58] that the incidence of cement embolic events regarding patients with IVC were significantly less than patients without cleft. Therefore, the IVC was regarded as a protective factor for cement embolic events.

\section{Conclusions}

The IVC sign is commonly observed in OVCF patients. Avascular necrosis theory is the most supported as a
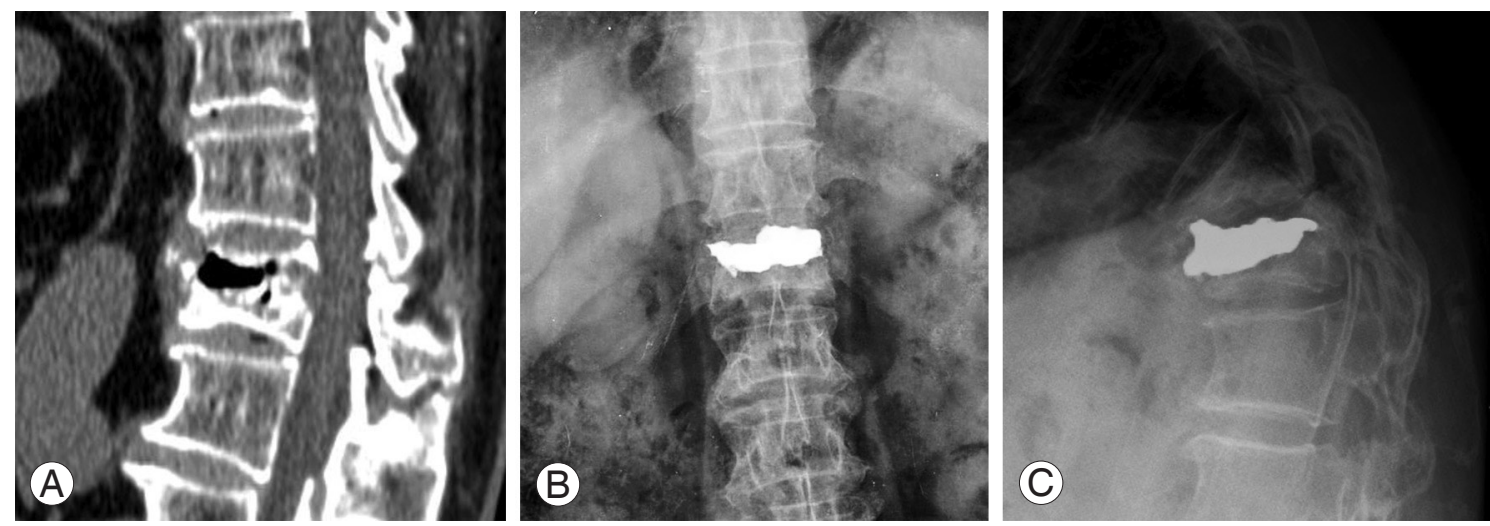

Fig. 4. A typical patient with intravertebral vacuum cleft and upper end-plate disrupt in our department (A), cement leak into intervertebral disk (B) and adjacent vertebral body fracture (C) at 3 months after the first surgery. 
pathogenesis of IVC sign. Biomechanics and osteoporosis are two other important contributors. The sign appears as a transverse, linear or semi-lunar radiolucent shadow in plain radiographs. The extension lateral view, CT scan and MR image were reported as more effective to detect the IVC sign. The Kümmell's disease with IVC sign reported by modern authors was incomplete consistent with the syndrome reported by Dr. Hermann Kümmell. However, no matter what etiologies cause vertebral compression fracture in patients with IVC sign, this sign is regarded as dynamic instability of the vertebral body that results in severe back pain. PVP can stabilize the vertebral body, relieve back pain, and restore vertebral body height and correct KA, and is thus recommended for these patients. PKP seems to be more effective at correction of $\mathrm{KA}$ and lower cement leakage in the limited literatures. Although the safety of PVP or PKP was reported, cement leakage into the intervertebral disk or pulmonary cement embolism should be taken cautiously.

\section{Conflict of Interest}

No potential conflict of interest relevant to this article was reported.

\section{References}

1. Francis RM, Aspray TJ, Hide G, Sutcliffe AM, Wilkinson P. Back pain in osteoporotic vertebral fractures. Osteoporos Int 2008;19:895-903.

2. Ehsanbakhsh AR, Akhbari H, Iraee MB, et al. The prevalence of undetected vertebral fracture in patients with back pain by dual-energy X-ray absorptiometry (DXA) of the lateral thoracic and lumbar spine. Asian Spine J 2011;5:139-45.

3. Kim DY, Lee SH, Jang JS, Chung SK, Lee HY. Intravertebral vacuum phenomenon in osteoporotic compression fracture: report of 67 cases with quantitative evaluation of intravertebral instability. J Neurosurg 2004;100:24-31.

4. Linn J, Birkenmaier C, Hoffmann RT, Reiser M, Baur-Melnyk A. The intravertebral cleft in acute osteoporotic fractures: fluid in magnetic resonance imaging-vacuum in computed tomography? Spine (Phila Pa 1976) 2009;34:E88-93.

5. Lane JI, Maus TP, Wald JT, Thielen KR, Bobra S, Luetmer PH. Intravertebral clefts opacified during vertebroplasty: pathogenesis, technical implications, and prognostic significance. AJNR Am J Neuroradiol 2002;23:1642-6.

6. McKiernan F, Jensen R, Faciszewski T. The dynamic mobility of vertebral compression fractures. J Bone Miner Res 2003;18:24-9.

7. Hasegawa K, Homma T, Uchiyama S, Takahashi H. Vertebral pseudarthrosis in the osteoporotic spine. Spine (Phila Pa 1976) 1998;23:2201-6.

8. Jang JS, Kim DY, Lee SH. Efficacy of percutaneous vertebroplasty in the treatment of intravertebral pseudarthrosis associated with noninfected avascular necrosis of the vertebral body. Spine (Phila Pa 1976) 2003;28:1588-92.

9. Maldague BE, Noel HM, Malghem JJ. The intravertebral vacuum cleft: a sign of ischemic vertebral collapse. Radiology 1978;129:23-9.

10. Theodorou DJ. The intravertebral vacuum cleft sign. Radiology 2001;221:787-8.

11. Sarli M, Perez Manghi FC, Gallo R, Zanchetta JR. The vacuum cleft sign: an uncommon radiological sign. Osteoporos Int 2005;16:1210-4.

12. Bhalla $S$, Reinus WR. The linear intravertebral vacuum: a sign of benign vertebral collapse. AJR Am J Roentgenol 1998;170:1563-9.

13. Kumpan W, Salomonowitz E, Seidl G, Wittich GR. The intravertebral vacuum phenomenon. Skeletal Radiol 1986;15:444-7.

14. Golimbu C, Firooznia H, Rafii M. The intravertebral vacuum sign. Spine (Phila Pa 1976) 1986;11:1040-3.

15. Chen LH, Lai PL, Niu CC, Chen CH, Chen WJ, Fu TS. Intracorporal bone grafting for vertebral compression fractures with intraosseous vacuum phenomenon. Int Orthop 2004;28:52-5.

16. Swartz K, Fee D. Kummell's disease: a case report and literature review. Spine (Phila Pa 1976) 2008;33:E1525.

17. Ratcliffe JF. The arterial anatomy of the adult human lumbar vertebral body: a microarteriographic study. J Anat 1980;131:57-79.

18. Van Eenenaam DP, el-Khoury GY. Delayed posttraumatic vertebral collapse (Kummell's disease): case report with serial radiographs, computed tomographic scans, and bone scans. Spine (Phila Pa 1976) 1993;18:1236-41.

19. Hermann G, Goldblatt J, Desnick RJ. Kummell disease: delayed collapse of the traumatised spine in 
a patient with Gaucher type 1 disease. Br J Radiol 1984;57:833-5.

20. Naul LG, Peet GJ, Maupin WB. Avascular necrosis of the vertebral body: MR imaging. Radiology 1989;172:219-22.

21. D'Ippolito G, Schiller PC, Ricordi C, Roos BA, Howard GA. Age-related osteogenic potential of mesenchymal stromal stem cells from human vertebral bone marrow. J Bone Miner Res 1999;14:1115-22.

22. Stabler A, Schneider P, Link TM, et al. Intravertebral vacuum phenomenon following fractures: CT study on frequency and etiology. J Comput Assist Tomogr 1999;23:976-80.

23. Armingeat T, Pham T, Legre V, Lafforgue P. Coexistence of intravertebral vacuum and intradiscal vacuum. Joint Bone Spine 2006;73:428-32.

24. Kummell H. Die rarefizierende ostitis der wirbelkorper. Deutsche Med 1895;21:180-1.

25. Kummell's disease. Lancet 1924;203:37.

26. Hosford J. Kummell's disease. Lancet 1936;227:249.

27. Steel HH. Kummell's disease. Am J Surg 1951;81:1617.

28. McKiernan F, Faciszewski T. Intravertebral clefts in osteoporotic vertebral compression fractures. Arthritis Rheum 2003;48:1414-9.

29. Brower AC, Downey EF Jr. Kummell disease: report of a case with serial radiographs. Radiology 1981;141:363-4.

30. Li KC, Li AF, Hsieh CH, Liao TH, Chen CH. Another option to treat Kummell's disease with cord compression. Eur Spine J 2007;16:1479-87.

31. Young WF, Brown D, Kendler A, Clements D. Delayed post-traumatic osteonecrosis of a vertebral body (Kummell's disease). Acta Orthop Belg 2002;68:13-9.

32. Wu MH, Huang TJ, Cheng CC, Li YY, Hsu RW. Role of the supine lateral radiograph of the spine in vertebroplasty for osteoporotic vertebral compression fracture: a prospective study. BMC Musculoskelet Disord 2010;11:164.

33. Malghem J, Maldague B, Labaisse MA, et al. Intravertebral vacuum cleft: changes in content after supine positioning. Radiology 1993;187:483-7.

34. Uetani M, Hashmi R, Hayashi K. Malignant and benign compression fractures: differentiation and diagnostic pitfalls on MRI. Clin Radiol 2004;59:124-31.

35. Kim YJ, Lee JW, Kim KJ, et al. Percutaneous verte- broplasty for intravertebral cleft: analysis of therapeutic effects and outcome predictors. Skeletal Radiol 2010;39:757-66.

36. Libicher M, Appelt A, Berger I, et al. The intravertebral vacuum phenomen as specific sign of osteonecrosis in vertebral compression fractures: results from a radiological and histological study. Eur Radiol 2007;17:2248-52.

37. Wang G, Yang H, Chen K. Osteoporotic vertebral compression fractures with an intravertebral cleft treated by percutaneous balloon kyphoplasty. J Bone Joint Surg Br 2010;92:1553-7.

38. Chen LH, Lai PL, Chen WJ. Unipedicle percutaneous vertebroplasty for spinal intraosseous vacuum cleft. Clin Orthop Relat Res 2005;(435):148-53.

39. Krauss M, Hirschfelder H, Tomandl B, Lichti G, Bar I. Kyphosis reduction and the rate of cement leaks after vertebroplasty of intravertebral clefts. Eur Radiol 2006;16:1015-21.

40. Ha KY, Lee JS, Kim KW, Chon JS. Percutaneous vertebroplasty for vertebral compression fractures with and without intravertebral clefts. J Bone Joint Surg Br 2006;88:629-33.

41. Peh WC, Gelbart MS, Gilula LA, Peck DD. Percutaneous vertebroplasty: treatment of painful vertebral compression fractures with intraosseous vacuum phenomena. AJR Am J Roentgenol 2003;180:1411-7.

42. Wiggins MC, Sehizadeh M, Pilgram TK, Gilula LA. Importance of intravertebral fracture clefts in vertebroplasty outcome. AJR Am J Roentgenol 2007;188:634-40.

43. Sun G, Jin P, Li M, Liu XW, Li FD. Height restoration and wedge angle correction effects of percutaneous vertebroplasty: association with intraosseous clefts. Eur Radiol 2011.

44. Lin CC, Chen IH, Yu TC, Chen A, Yen PS. New symptomatic compression fracture after percutaneous vertebroplasty at the thoracolumbar junction. AJNR Am J Neuroradiol 2007;28:1042-5.

45. Teng MM, Wei CJ, Wei LC, et al. Kyphosis correction and height restoration effects of percutaneous vertebroplasty. AJNR Am J Neuroradiol 2003;24:1893900 .

46. Goz V, Koehler SM, Egorova NN, et al. Kyphoplasty and vertebroplasty: trends in use in ambulatory and inpatient settings. Spine J 2011;11:737-44.

47. Belkoff SM, Mathis JM, Fenton DC, Scribner RM, 
Reiley ME, Talmadge K. An ex vivo biomechanical evaluation of an inflatable bone tamp used in the treatment of compression fracture. Spine (Phila Pa 1976) 2001;26:151-6.

48. Leidig-Bruckner G, Minne HW, Schlaich C, et al. Clinical grading of spinal osteoporosis: quality of life components and spinal deformity in women with chronic low back pain and women with vertebral osteoporosis. J Bone Miner Res 1997;12:663-75.

49. Schlaich C, Minne HW, Bruckner T, et al. Reduced pulmonary function in patients with spinal osteoporotic fractures. Osteoporos Int 1998;8:261-7.

50. Ismail AA, Cockerill W, Cooper C, et al. Prevalent vertebral deformity predicts incident hip though not distal forearm fracture: results from the European Prospective Osteoporosis Study. Osteoporos Int 2001;12:85-90.

51. Nieuwenhuijse MJ, Van Erkel AR, Dijkstra PD. Cement leakage in percutaneous vertebroplasty for osteoporotic vertebral compression fractures: identification of risk factors. Spine J 2011;11:839-48.

52. Tanigawa N, Kariya S, Komemushi A, et al. Cement leakage in percutaneous vertebroplasty for osteoporotic compression fractures with or without intravertebral clefts. AJR Am J Roentgenol 2009;193:W442-5.

53. Ren H, Shen Y, Zhang YZ, et al. Correlative factor analysis on the complications resulting from cement leakage after percutaneous kyphoplasty in the treatment of osteoporotic vertebral compression fracture. J Spinal Disord Tech 2010;23:e9-15.

54. Komemushi A, Tanigawa N, Kariya S, et al. Percutaneous vertebroplasty for osteoporotic compression fracture: multivariate study of predictors of new vertebral body fracture. Cardiovasc Intervent Radiol 2006;29:580-5.

55. Lin EP, Ekholm S, Hiwatashi A, Westesson PL. Vertebroplasty: cement leakage into the disc increases the risk of new fracture of adjacent vertebral body. AJNR Am J Neuroradiol 2004;25:175-80.

56. Krueger A, Bliemel C, Zettl R, Ruchholtz S. Management of pulmonary cement embolism after percutaneous vertebroplasty and kyphoplasty: a systematic review of the literature. Eur Spine J 2009;18:1257-65.

57. Yoo KY, Jeong SW, Yoon W, Lee J. Acute respiratory distress syndrome associated with pulmonary cement embolism following percutaneous vertebroplasty with polymethylmethacrylate. Spine (Phila Pa 1976) 2004;29:E294-7.

58. Kim YJ, Lee JW, Park KW, et al. Pulmonary cement embolism after percutaneous vertebroplasty in osteoporotic vertebral compression fractures: incidence, characteristics, and risk factors. Radiology 2009;251:250-9. 